\title{
Source credibility in social media: A case study of a reddit community
}

\author{
Amy Hartzell, Robert Morris University, amykhartzell@gmail.com \\ Beau Jackson, Robert Morris University,jackson.beau.michael@gmail.com \\ Jamie Pinchot, Robert Morris University, pinchot@rmu.edu
}

\begin{abstract}
This exploratory case study focused on understanding how source credibility is established in social media. It analyzed posts from the r/wallstreetbets Reddit community during spring of 2021 when this community was highlighted in the media for its impact on stock trading. The study found that the subreddit established source credibility in a variety of ways that are unique to Reddit amongst social media platforms. Initial credibility was represented by the popularity (number of followers) of the community, the use of volunteer moderators, and the presence of shared language elements known only to members. Transactional credibility was represented through the use of upvotes and downvotes to determine which content is seen by users in the news feed, and also through the use of awards that are given to users who have made meaningful posts or contributions. Terminal credibility was determined for users and posts over time as upvotes and awards were accumulated within the community, serving to enhance the reputation of users who received them. Additionally, subreddit communities themselves were found to attain terminal credibility via reputation earned from the shared culture and content of the community. Discussion of the findings and opportunities for future research are presented.
\end{abstract}

Keywords: social media, credibility, online credibility, source credibility, online communities, Reddit

\section{Introduction}

Approximately four billion social media users worldwide contribute content through the various social media platforms (Windham-Bradstock, 2020). With the ubiquitous usage of social media technology, it can be challenging for social media users to ascertain if online content is credible. Credibility is the quality of being believed or trusted by others (Anupam et al., 2021).

Today, the Internet represents a primary way that many people stay informed. Therefore, credibility of online sources, including social media, are of critical importance. In the past, offline information was vetted through a variety of well-established media organizations including newspapers, magazines, radio, and television broadcasts, as well as private companies, non-profit organizations, and information intermediaries such as experts and opinion leaders. The reputation of each of these traditional media channels, organizations, or experts could be used to reduce uncertainty about the credibility of information provided. However, in the digital world, the assessment of credibility is far more complex (Viviani \& Pasi, 2017). Digital media have in many ways shifted the burden of evaluating online information from professional gatekeepers to individual information consumers (Flanagin \& Metzger, 2008). 


\section{Issues in Information Systems}

Volume 22, Issue 3, pp. 212-223, 2021

Social media sites, in particular, include user generated content that is shared or contributed by a wide range of users and sources. Some social media sites also allow posters to be anonymous (or to use aliases rather than real names). Due to containing content created by this multiplicity of sources, social media sites also often lack clarity in the context of information shared. The user generated content on social media sites is able to be published with little to no forms of external control (Viviano \& Pasi, 2017). In addition, algorithms used by social media sites often attempt to tailor content for individual users based on content they have previously read and liked. Over time, these types of algorithms and content filtering can turn social media sites into "palaces of mirrors in which everyone seeks and finds possible confirmations to their opinions and reflections" (Viviano \& Pasi, 2017, p. 2). This effect is also known as a "filter bubble" (Pariser, 2011) or the "echo chamber phenomenon" of social media (Quattrociocchi et al., 2016), where each user sees only personalized sets of content rather than the entire picture, often leading to severe misunderstanding of the facts or truth of a situation. All of these factors combine to create a complex digital landscape of information where the ability to discern credible content from misinformation becomes increasingly difficult.

This study addresses the complex issue of credibility in social media through an exploratory case study of one social media site, Reddit. Reddit is a social media site comprising more than one million communities and over 168 million unique users (Widman, 2021). These communities are essentially collections of discussion forums known as subreddits. Each subreddit represents a different topic, and online users share news, information, opinions, and other sentiments via posts in the Subreddit and comments on other users' posts. Popularly known as the "front page of the Internet", the site has been labeled as the seventh most popular social media site in the United States and eighteenth most popular worldwide (Widman, 2021, para. 2). While news organizations can share content directly to Reddit, the site is known for its active base of users, known as Redditors, who act as citizen journalists by sharing, reposting, and recirculating media content (Kilgo et al., 2016). While other social media sites have online users who boastfully share their identity, Reddit users are semi-anonymous (Widman, 2021). Reddit differs from other platforms such as Facebook, Twitter, or Instagram as they only require a username as an identifier and users often do not disclose their real identity. Despite the semi-anonymity of Redditors, the platform has developed and built a culture of community and authenticity.

This study will address one primary research question focused on understanding the nuances of credibility in online social media sites, and in particular in the Reddit community:

RQ1: How is source credibility established in Reddit communities?

Due to its popularity, reputation for citizen journalism, unique online community characteristics, and the fact that it has not been as widely studied as other social media sites such as Facebook and Twitter, Reddit is a good candidate for an exploratory study focused on understanding social media credibility.

\section{Literature Review}

Credibility in social media has been more widely studied in the two most popular social media sites, Twitter and Facebook. Mitra and Gilbert (2015) conducted real-time tracking of more than 60 million tweets with human annotators over a period of three months and found that $24 \%$ of the events in the global tweet stream are not perceived as credible. Westerman, Spence, and Van Der Heide (2014) studied credibility in Twitter posts and found that the recency of tweets impacted the participants' perception of credibility. In addition, several studies have developed models to determine or automate credibility ratings for tweets (Setiawan et al., 2020; Hassan et al., 2019; Yang et al., 2019; Buntain \& Golbeck, 2017). 


\section{Issues in Information Systems}

Volume 22, Issue 3, pp. 212-223, 2021

Research on Facebook has found that a major credibility issue relates to the algorithms used to determine what news content is shown to a particular user. Quattrociocchi et al. (2016) found that echo chambers do exist within Facebook. They found that aggregation of favored information reinforces selective exposure to information and incites polarization between groups of Facebook users with differing views on specific topics. In addition, Borah and Xiao (2018) studied perceptions of health information on Facebook and found that posts from an expert source, with a positive message framing and high number of likes was considered the most credible message. A number of studies have also looked at credibility of individual Facebook posts, and have attempted to develop automated tools to aid users in determining credibility (Idrees et al., 2019; Saikaew \& Noyunsan, 2015).

In contrast, the Reddit community has primarily been studied in terms of understanding community engagement behaviors (Staudt Willet \& Carpenter, 2020; Record et al., 2018; Horne et al., 2017). There has not been a focus in literature on understanding the factors that contribute to the credibility of posts in Reddit communities. This study will contribute to the literature by addressing this gap.

\section{Types of Credibility in Social Media}

Credibility can be interpreted in different ways by social media users. Online users may find content to be credible if the author appears to have integrity, is trustworthy, reliable, authentic, committed, highly regarded, and has a positive reputation (Jung-Kuei \& Yi-Jin, 2020). Additionally, studies have discovered social media users may be influenced more by the emotional impact or attached personal belief than the objective truth. This is known as post-truth (Anupam et al., 2021). Credibility in social media is described in a variety of ways in the literature. Some primary credibility constructs include source credibility, medium credibility, and message or content credibility (Buhlmann \& Gisler, 2006).

Source credibility refers to the trustworthiness and likelihood of credibility from a particular source or outlet on social media (Buhlmann \& Gisler, 2006). Source credibility may be determined by the level of perceived authenticity of the author, and past reviews or ratings attached to the author's profile. Positive perceptions of a message in an online environment can be quantified by using a scale of measurement such as believability, fairness, accuracy, and reliability (Jung-Kuei \& Yi-Jin, 2020). Source Credibility Theory refers to the receiver's perception of the credibility of a message source. Source credibility has a large impact on the persuasiveness of a message (Buhlmann \& Gisler, 2006). People tend to believe and accept messages from highly credible sources. This belief is derived from the perception of the message receivers inferring that credibility implies truth causing users to believe that a message from a more credible source is more valid (Buhlmann \& Gisler, 2006). In addition, Jung-Kuei and Yi-Jin (2020) posit that content from a credible source positively influences the information receiver's attitude and consequent behaviors.

Medium credibility is the perceived level of credibility users have for a particular social media platform ((Buhlmann \& Gisler, 2006)). Online users may have a perception variance of credibility for the different types of social media tools. For example, the purpose of TikTok is more entertainment-centric, whereas LinkedIn is a professional networking tool. In comparison, social media users may find LinkedIn to be more credible based upon the content shared.

Message or content credibility refers to the accuracy of content shared and the quality of information. This type of credibility refers to how specific content resonates with social media users. The perception of the validity of the online content is an important factor to consider when evaluating message or content 


\section{Issues in Information Systems}

Volume 22, Issue 3, pp. 212-223, 2021

credibility. Users will assess the quality of the content based upon the author's reputation, previous content shared, and the feedback from other online users (Buhlmann \& Gisler, 2006).

\section{Social Media Credibility Phases}

As social media users analyze and assess if the content is credible on social media platforms, there are three stages or phases when credibility is defined: (a) initial credibility, (b) transactional credibility, and (c) terminal credibility (Jung-Kuei \& Yi-Jin, 2020).

\section{Initial Credibility}

Initial credibility is the first phase when credibility is established. This initial interaction is when credibility is assumed of the author. The user may base their subjective opinion contingent upon previous content the author has shared or the existing projection of credibility from other users. If the content author has a credible presence, the social media user may assume the context will be accurate, trustworthy, and believable. If the author does not have an existing credible and known presence, then the initial credibility will be low. Online users will make assumptions and judgments according to the knowledge and evidence they have (Jung-Kuei \& Yi-Jin, 2020).

\section{Transactional Credibility}

The second phase is transactional credibility, and this action occurs after the initial credibility has been created, and during the engagement process on social media between the social media users and the author. The social media user most likely will examine and analyze the competence, likeability, character, and professionalism of the message. If the content author maintains credibility, builds trust through sincere and authentic content, then the transactional credibility increases (Jung-Kuei \& Yi-Jin, 2020).

\section{Terminal Credibility}

The last phase is terminal credibility, and this is the perception the social media user has when the interaction is completed. If the social media user has positive experiences and perceives the content as credible, then the terminal credibility will be high. The author can cumulatively build rapport and create connection through consistency and fostering a relational connection with the social media user. This final form of credibility is also influenced by facts and evidential points of reference. If this interaction is not positive, it will impact the initial credibility factor during the next experience with the author (JungKuei \& Yi-Jin, 2020).

\section{Methodology}

An exploratory case study method was used to analyze one specific case involving a community on Reddit. The subreddit that was studied was the wallstreetbets subreddit (denoted on the Reddit site as $\mathrm{r}$ /wallstreetbets). This subreddit was chosen due to its prominence in the news media in a controversial event, as well as for its popularity and high number of users. As part of the case study, 46 threads (These threads contained between 23,000 - 97,500 comments) in the r/wallstreetbets subreddit, that were posted in January and February 2020, were collected, read, and analyzed for distinctive features that impacted source credibility, using the construct of source credibility as a lens for analysis. 


\section{Issues in Information Systems}

Volume 22, Issue 3, pp. 212-223, 2021

\section{The Case: r/wallstreetbets}

In October 2020, Reddit's r/wallstreetbets had approximately 1.55 million members within its community (Widman, 2021). This subreddit is a community where users can share information and opinions about the stock market. In early 2021, this subreddit received a great deal of news coverage because members of the subreddit allegedly facilitated an organized movement against major hedge fund companies involving stock trades of GameStop (GME) stock (Karabell, 2021). Six months later, and after the initial media coverage of the GameStop (GME) stock phenomenon, r/wallstreetbets exceeded 9.8 million members (Widman, 2021).

Through the viral power and influence of the r/wallstreetbets community members, these users chose to follow the consensus of the community and engaged in a highly speculative and high-risk decision to invest in GME. Many eyebrows were raised from January through February of 2021 (Thorbecke, 2021) as the share price for GME went from $\$ 17.37$ on January 4th to as high as $\$ 483$ on January 28th (NYSE, 2021). Conflicts of interest between retail trading software company, Robinhood, and securities trading company, Citadel Securities, were revealed when Robinhood cut retail traders off from trading GME stocks (as well as a few others) while still allowing hedge fund companies, like Citadel, to continue trading (MacMillan, 2021). In an effort to protect retail traders, the Securities Exchange Commission (SEC) prompted an investigation into the relationship (Franck, 2021) and a Congressional hearing was held by the House Financial Services Committee which included the Robinhood CEO, Citadel CEO, Melvin Capital CEO, Reddit CEO, a financial regulation expert from the Cato Institute, and a retail investor (Kailath, 2021). The situation made headlines across every major media channel with each channel sharing their thoughts about how it all happened, or rather, how it was possible. Many pointed to the social media platform, Reddit, as having been the facilitator of an organized movement against major hedge fund companies and a potential accessory to mass market manipulation on a global scale (Karabell, 2021).

\section{Results}

The posts involved in the case study were analyzed through the lens of source credibility. In particular, we provide an analysis of how the three phases of initial credibility, transactional credibility, and terminal credibility are exemplified in the $r$ /wallstreetbets case. Specific examples are provided to describe the social media user experience and demonstrate the credibility patterns developed within each phase.

\section{Initial Credibility in r/wallstreetbets}

Initial credibility occurs when credibility is established prior to the interaction between the user and the source. Illustrated within Reddit's communities are three examples of how initial credibility may be gained on its platform with the moderators, language, and membership numbers. Moderators are Reddit members who volunteer to establish and monitor the community. They decide the subject matter and branding. They supervise post behavior and protect against spammers (What's a moderator?, 2021). Reddit provides moderator guidelines that, if followed, will enhance the initial credibility of the group. The guidelines are as follows: engage in good faith, provide a community description, establish clear, concise, and consistent guidelines, have a stable and active team, provide proper branding, engage in email communication, allow appeals to moderator responses, abide by content policy, and respect the platform. Following these guidelines provides for a positive user experience which adds to initial credibility (Moderator Guidelines, 2017).

Similar to real life, each community has its own way of communicating - often having its own use of language or restricted code. Restricted code is a "style of language use associated with informal situations, 


\section{Issues in Information Systems}

Volume 22, Issue 3, pp. 212-223, 2021

characterized by linguistic predictability and by its dependence on the external context and on the shared knowledge and experience of the participants for conveying meaning" (Kilgo, et al., 2016, para. 6). Consequently, when someone uses the platform within this subreddit, initial credibility is gained if and only if the user can understand and use the appropriate language when posting and responding. For example, consider the underlined terms in the following comment posted by u/uwillmire on January 28, 2021 at 11:30am EST:

Buy more during dips if you can, but at least hold.

We just have to hold until they fold. Today's actions by several brokers just show how desperate the hedge funds are getting.

Hold with your immovable diamond hands for all that you hold dear and we will be breaking Wall Street TOGETHER while making gargantuan tendies in the end!

In the post above, "dips" refers to when the price of stock has dropped to its perceived low point before increasing in value. The word "hold" refers to maintaining one's position on a stock - not selling as to maintain its value. The phrase "diamond hands" refers to someone who is able to resist selling stock owned even as they watch its value drop drastically and consequently pass on making their money back in hopes the stock reverses its trend and increases in value. These are only a few examples of the restricted code used in the $\mathrm{r}$ /wallstreetbets community.

The last illustration of initial credibility within this case study is correlated with the size of the community. Jin and Phua (2014) found a higher number of followers increased source credibility. In January 2020, Reddit's r/wallstreetbets had approximately 775,000 members within its community. In October, ten months later, the number doubled to 1.55 million. Six months later and after the media coverage of the GME stock phenomena, r/wallstreetbets exceeded 9.8 million members (Subreddit Stats, 2021). Overnight it became one of the most popular places to discuss securities trading, which increased initial credibility dramatically.

\section{Transactional Credibility in r/wallstreetbets}

Transactional credibility occurs after the initial credibility has been created, and during the engagement process on social media between the social media user and the author. In Reddit communities, users have the ability to interact with posts by voting them up or down using buttons located at the bottom of each post. As posts are voted on (up or down), they are moved (up or down) the news feed of the community. As more upvotes accumulate, the post continues to move to the top of the news feed and the more often the post is seen, by more users. Any member within the group can vote (either up or down). The following post by $\mathrm{u} /$ vrweensy on January 28, 2021 at 8:40am EST is a perfect example of how this feature may be used as a form of transactional credibility in reference to purchasing GME stock. Note that asterisks were added to the quote below by the authors to redact explicit language:

\section{UPVOTE so everyone sees we got SUPPORT}

Robinhood legitimately hid \$NOK \$AMC \$GME and \$NAKD from search. Internet psychology 101, this will only make things worse for you Wall Street. Things are getting blown the **** up today. Take all the cash you can afford to lose and buy buy buy. 


\section{Issues in Information Systems}

Volume 22, Issue 3, pp. 212-223, 2021

This was the second most popular vote (at the time of this writing) for 2021 with over 316,000 upvotes. Transactional credibility is built by the members of the community for both the user who created the post, as well as the post itself. Market mavens are "[i]ndividuals who act as hubs, gatherers and disseminators of general market information, and who have a perceived high level of credibility and expertise (Humphreys, 2016, p. 163). The market mavens of $r$ /wallstreetbets diffused the idea of a financial benefit within the community, consequently persuading millions of users to further invest in GME stock. The implications of $r$ wallstreetbets community members having high levels of influence within the community but limited professional financial knowledge resulted in the loss of life savings, jobs, and financial stability (Chalk \& Messner, 2021).

Another mechanism in the subreddit that impacted transactional credibility was the use of awards. Users may purchase and give awards to contributors for their posts. Awards are purchased by coins - virtual money that may be bought with real money. Awards come in four main types: silver, gold, platinum, and community. Silver awards cost 100 coins and are just status symbols for the user. Gold awards cost 500 coins and give the recipient a week of Reddit Premium (the paid version of membership) along with 100 coins. Platinum awards cost 1800 coins and give the recipient a month of Reddit Premium and 700 coins (Coins, 2021). Community awards vary by each subreddit or community. They are made by the moderators who choose the price, name, and icon of the award. Ultimately, the awards are given to those contributors with exemplary posts and contributions (Can someone explain awards to me?, 2019; Coins, 2021). For example, a post made by u/Deep*******Value (asterisks added by the authors to redact explicit language) received over 8,300 awards. Of those awards, the user received five "Ternion All-Powerful Awards" which each cost 50,000 Reddit coins. This is the most prestigious and expensive award on Reddit (Ternion AllPowerful Award, 2021). Notably the author of this post was also the individual who attended the Congressional hearing and is considered the most credible and original source for starting the GME buying trend.

\section{Terminal Credibility in r/wallstreetbets}

The last phase is terminal credibility, and this is the perception the social media user has when the interaction is completed. The key idea is the cyclical nature of source credibility. Initial and transactional credibility add to the culture that enhances (or discourages) user experience. Given Reddit's approach to encourage subreddit communities to develop their own cultures, members join and leave as they identify with the different communities. The moderators, the language, the size, the voting, and the award system all play a part in contributing to the user's experience. Consider the following post by $\mathrm{u} / \mathrm{Lunar}$ on January 30, 2021 at 8:04pm EST:

I am proud to do my part in paying forward our good fortune with a donation of 6 Nintendo Switches and games to go with them to the Children's Minnesota Hospital. Cant Stop. Won't Stop. GameStop. (Still long 50 shares I WILL NOT SELL)

In the end, not only do users and posts gain credibility via upvotes and awards, but the communities of culture embodied by each subreddit are also increasing their own credibility. This credibility is noticed by outsiders and begins to have an impact on other communities as well. GME and Meme stocks grew exponentially overnight driven by the credibility of the community members; a GME member was asked to participate in the Congressional hearing regarding the use and misuse of short selling by large hedge funds and app developers; and r/wallstreetbets gained over 1.5 million members overnight (Gosh, 2021). 


\section{Issues in Information Systems}

Volume 22, Issue 3, pp. 212-223, 2021

\section{Discussion}

Source credibility refers to the trustworthiness and likelihood of credibility from a source on social media. Viewed through the lens of source credibility, it is clear that the Reddit community, and in particular, the subreddit $r$ /wallstreetbets, establishes source credibility in a variety of ways that are unique to Reddit amongst social media platforms. This exploratory case study found that initial credibility is established in Reddit through the popularity of the subreddit community, which is in keeping with Jin and Phua's (2014) findings about Reddit communities and also parallels Borah and Xiao's (2018) finding in their study of Facebook posts that a higher number of likes increased credibility. Additionally, the use of moderators (volunteers) within the community and the presence of a shared language or restricted code known only to members of the community also established initial credibility.

Transactional credibility was represented in the case through the use of upvotes and downvotes to determine the order in which content is seen by users, and also through the use of awards that are given to users who have made meaningful posts or contributions. Awards are given by other users and are purchased with virtual coins that are in turn purchased with real money. Thus, transactional credibility in the subreddit is supported by real money offered from one user to another in appreciation of the content provided to the community.

Finally, terminal credibility is determined for users and posts over time as upvotes and awards are accumulated within the community, serving to enhance the reputation of users who receive them. In addition, terminal credibility can also be seen for subreddit communities themselves, as evidenced by the overall media attention received by the r/wallstreetbets community. The culture of each subreddit community, including its shared restricted code and language, as well as its individual characteristics, moderators, and community awards, contributes to the overall reputation of the community. It is clear from this case study that a subreddit is capable of impacting communities outside of its own, and indeed, outside of Reddit itself.

\section{Limitations}

This exploratory case study served to analyze the impact of source credibility in this Reddit community, but it has truly only scratched the surface of this topic. The study was limited by the theoretical lens chosen for the analysis, and also by the timeframe and subreddit community that was chosen. There is a wealth of data still to study in the Reddit community, which stands apart from other social media sites in its unique use of volunteer moderators, upvotes as well as downvotes (most other sites only allow for the equivalent of upvotes such as "likes"), awards, and a shared common language within its communities.

\section{Future Research}

In exploring the different facets of how social media users may define content as credible, three specific avenues for further research have emerged from this exploratory study. First, the shared language or restricted code that is used within some Reddit communities is extremely unique to this social media site and warrants further investigation. It could be useful to study the perceptions of Reddit users in regard to this shared language.

Second, additional research could be conducted to explore both social and cultural capital within Reddit communities. Humphreys (2016) defines cultural capital as the "articulation of tastes, embodied practices, or institutionalized formal education that constitutes a resource in the reproduction or advancement of social class" (p. 285). Reddit is a great example of a platform where cultural capital may be established between 


\section{Issues in Information Systems}

Volume 22, Issue 3, pp. 212-223, 2021

the moderator, members, and within the communities. Additional research can be conducted to explore how cultural capital may be created through the exchanges in the subreddit communities, and the impact it may have on internal and external communities and social media users.

Finally, research has shown that echo chambers or filter bubbles exist in social media sites such as Facebook and Twitter (Pariser, 2011; Quattrociocchi et al., 2016) where algorithms control the content that is shown to users. In contrast, Reddit uses volunteer moderators and upvotes and downvotes to control the stream of content that is delivered to the news feed. This difference warrants further study to see if it alleviates the issue of the social media echo chamber.

\section{Conclusion}

This case study in Reddit is an illustration of how source credibility exists in social media and may be facilitated within online environments by the "non-experts" (such as social media users), as opposed to traditional academic environments (such as journals and other published works). It is also an example of how source credibility provides a great deal of power to social media platforms to influence other communities, of both the digital and in-person varieties.

\section{References}

Anupam, D., Dongsong Z., \& Lina Z. (2021). Personalized ranking of online reviews based on consumer preferences in product features. International Journal of Electronic Commerce, $25(1)$, pp. $29-50$.

Borah, P., \& Xiao, X. (2018). The importance of 'likes': The interplay of message framing, source, and social endorsement on credibility perceptions of health information on Facebook. Journal of Health Communication, 23(4), 399-411.

Buhlmann, H., \& Gisler, A. (2006). A Course in Credibility Theory and its Applications. Springer Publishing

Buntain, C., \& Golbeck, J. (2017). Automatically identifying fake news in popular Twitter threads. IEEE International Conference on Smart Cloud, 208-215.

Can someone explain awards to me? (2019, August 30). Reddit. https://www.reddit.com/r/help/comments/cxnftb/can_someone_explain_awards to me/

Chalk, A. \& Messner, S. (2021). After GameStop's share price plummets, Reddit's WallStreetBets investors are hurting. PC Gamer. After GameStop's share price plummets, Reddit's $\underline{\text { WallStreetBets investors are hurting | PC Gamer }}$

Coins. (2021). Reddit. https://www.reddit.com/coins

Franck, T. (2021, January 29). SEC reviewing volatility amid GameStop frenzy, vows to protect retail investors. CNBC. https://www.cnbc.com/2021/01/29/sec-reviewing-recent-trading-volatilityamid-gamestop-frenzy-vows-to-protect-retail-investors.html

Flanagin, A.J., \& Metzger, M.J. (2008). Digital media and youth: Unparalleled opportunity and unprecedented responsibility. MacArthur Foundation Digital Media and Learning Initiative. 


\section{Issues in Information Systems}

Volume 22, Issue 3, pp. 212-223, 2021

Ghosh, S. (2021). Reddit group WallStreetBets hits 6 million users overnight after a wild week of trading antics. Business Insider. https://www.businessinsider.com/wallstreetbets-fastest-growingsubreddit-hits-58-million-users-2021-1

Hassan, N.Y., Gomaa, W.H., Khoriba, G.A., \& Haggag, M.H. (2019). Credibility detection in Twitter using word n-gram analysis and supervised machine learning techniques. International Journal of Intelligent Engineering \& Systems, 13(1), 291-300.

Horne, B.D., Adali, S., \& Sikdar, S. (2017). Identifying the social signals that drive online discussions: A case study of Reddit communities. In 2017 IEEE $26^{\text {th }}$ International Conference on Computer Communication and Networks, 1-9.

Humphreys, A. (2016). Social media: Enduring principles. Oxford University Press.

Idrees, A.M., Alsheref, F.K., \& ElSeddawy, A.I. (2019). A proposed model for detecting Facebook news' credibility. International Journal of Advanced Computer Science and Applications, 10(7), 311316.

Jin, S.A., \& Phua, J. (2014). Following celebrities' tweets about brands: The impact of Twitterbased electronic word-of-mouth on consumers' source credibility perception, buying intention, and social identification with celebrities. Journal of Advertising, 43(2), 181-195.

Jung-Kuei, H. \& Yi-Jin, L. (2020). Will you ever trust the review website again? The importance of source credibility. International Journal of Electronic Commerce, 24(2), 225-275. https://doi.org/10.1080/10864415.2020.1715528

Kailath, R. (2021, February 18). 'I am not a cat ... I am not a hedge fund': Trader roaring kitty tells congress. NPR.org. https://www.npr.org/2021/02/18/968829355/gamestop-hearing-todayroaring-kitty-along-with-ceos-to-appear-before-congress

Karabell, Z. (2021, January 28). How the GameStop trading surge will transform Wall Street. Time. https://time.com/5934285/gamestop-trading-wall-street/

Kilgo, D., Yoo, J., Sinta, V., Geise, S., Suran, M., \& Johnson, T. (2016). Led it on Reddit: An exploratory study examining opinion leadership on Reddit. First Monday, 21(9). http://dx.doi.org/10.5210/fm.v21i9.6429

MacMillan, D. (2021, January 29). Robinhood and Citadel's relationship comes into focus as Washington vows to examine stock-market moves. Washington Post; The Washington Post. https:/www.washingtonpost.com/business/2021/01/29/robinhood-citadel-gamestop-reddit/

Mitra, T., \& Gilbert, E. (2015). CREDBANK: A large-scale social media corpus with associated credibility annotations. Proceedings of the Ninth International AAAI Conference on Web and Social Media, 258-267.

Moderator Guidelines for Healthy Communities. (2017). Reddit. https://www.redditinc.com/policies/moderator-guidelines-for-healthy-communities 


\section{Issues in Information Systems}

Volume 22, Issue 3, pp. 212-223, 2021

NYSE. (2021). Nyse.com. https://www.nyse.com/quote/XNYS:GME

Quattrociocchi, W., Scala, A., \& Sunstein, C.R. (2016). Echo chambers on Facebook. SSRN. http://ssrn.com/abstract=2795110

Rahman, M.H., Prama, T.T., \& Anwar, M.M. (2020). Modeling topic specific credibility in Twitter based on structural and attribute properties. HIS, 580-589.

Record, R.A., Silberman, W.R., Santiago, J.E., \& Ham, T. (2018). I sought it, I Reddit: Examining health information engagement behaviors among Reddit users. Journal of Health Communication, 23(5), 470-476.

Saikaew, K.R., \& Noyunsan, C. (2015). Features for measuring credibility on Facebook information. International Journal of Computer, Control, Quantum and Information Engineering, 9(1), 174177.

Setiawan, E. B., Widyantoro, D. H., \& Surendro, K. (2020). Measuring information credibility in social media using combination of user profile and message content dimensions. International Journal of Electrical and Computer Engineering, 10(4), 3537-3549.

Staudt Willet, K.B., \& Carpenter, J.P. (2020). Teachers on Reddit? Exploring contributions and interactions in four teaching-related subreddits. Journal of Research on Technology in Education, $52(2), 216-233$

Subreddit Stats - statistics for every subreddit. (2021). Subredditstats.com. https://subredditstats.com/r/wallstreetbets

Ternion all-powerful award. (2021). Fandom. https://reddit.fandom.com/wiki/Ternion_AllPowerful_Award

Thorbecke, C. (2021, February 13). GameStop timeline: A closer look at the saga that upended Wall Street. ABC News; ABC News. https://abcnews.go.com/Business/gamestop-timeline-closer-sagaupended-wall-street/story?id $=75617315$

Viviani, M., \& Pasi, G. (2017). Credibility in social media: opinions, news, and health information - a survey. WIREs Data Mining and Knowledge Discovery, 7(5), 1-25. doi: 10.1002/widm.1209

Westerman, D., Spence, P., \& Van Der Heide, B. (2014). Social media as information source: Recency of updates and credibility of information. Journal of Computer-Mediated Communication, 9, 171183.

What's a moderator? (2021). Reddit. https://reddit.zendesk.com/hc/en-us/articles/204533859-What-is-amoderator-

\#: : text $=\mathrm{A} \% 20$ moderator $\% 20$ is $\% 20$ just $\% 20$ a,logo $\% 20$ and $\% 20$ styling $\% 2 \mathrm{C} \% 20 \mathrm{if} \% 20$ any

Widman, J. (2021). What is Reddit? Digital Trends.

https://www.digitaltrends.com/web/what-is-reddit/ 


\section{Issues in Information Systems}

Volume 22, Issue 3, pp. 212-223, 2021

Windham-Bradstock, C. (2020). The do's and don'ts of social media etiquette at work. Forbes. https://www.forbes.com/sites/forbeshumanresourcescouncil/2020/04/03/the-dos-anddonts-of-social-media-etiquette-at-work/?sh=1ce41 eb64c40

Yang, J., Yu, M., Qin, H., Lu, M., \& Yang. C. (2019). A Twitter data credibility framework - Hurricane Harvey as a use case. ISPRS International Journal of Geo-Information, 8(3), 111. 